\title{
THE ALPHA-EFFECT BY SUPERNOVA EXPLOSIONS
}

\author{
M. KAISIG ${ }^{1}$, G. RÜDIGER ${ }^{1,2}$, H.W. YORKE ${ }^{1}$
}

${ }^{1}$ Institut für Astronomie und Astrophysik der Universität Würzburg

${ }^{2}$ Astrophysikalisches Institut Potsdam

ABSTRACT. The turbulence in galaxies is assumed to be driven by a sample of stochastically occurring supernova explosions. For simplicity, we replace the complicated real flow by the velocity field of the explosions themselves. The resulting turbulence model can be used to determine the turbulent electromotive force, $\left\langle\mathbf{u}^{\prime} \times \mathbf{B}^{\prime}\right\rangle$, a basic quantity in the dynamo theory.

If the distribution function of the SN explosion rate is known, it is sufficient to compute the $\alpha$-effect for a single explosion in a rotating, density stratified galaxy. We use an axisymmetric simulation code to determine the helical velocity field of the explosion, and consider only explosions located on the rotation axis. The (homogeneous) magnetic field is assumed to be purely vertical. Thercforc, only the $\alpha_{z z}$-component of the $\alpha$-tensor can be obtained with this method.

The induced magnetic field fluctuations must be correlated with the velocity field in order to get the turbulent electromagnetic force. The resulting $\alpha$-effect for a sample of explosions starting in the galactic midplane possesses a $\mathrm{z}$-profile with an amplitude of about $60 \mathrm{~m} / \mathrm{s}$. It is always negative in the northern hemisphere and positive in the southern hermisphere. The scale of the $\alpha$-profile is about $60 \mathrm{pc}$.

The influence of the density distribution proved to be small. We consider the extreme case of uniform explosion rate and find $\alpha$-values of only few meters per second for a density scale height of $100 \mathrm{pc}$.

\section{Introduction}

The generation of magnetic fields on astrophysical scales remains one of the most challenging problems in astrophysics. It is widely believed that the origin of the galactic magnetic field results from the amplification of a seed field throught dynamo action. In the dynamo theory, the large scale magnetic field is sheared in the azimuthal direction by differential rotation about the galactic center, and the radial field is regenerated from the azimuthal field through turbulence (the socalled $\alpha$-effect). In contrast to the large scale differential rotation, whose properties are reasonable well established, the $\alpha$-effect is still poorly understood.

This paper is devoted to the determination of the $\alpha$-parameter in the galaxy by means of numerical simulations. We assume that the main source of turbulence in the interstellar medium is provided by supernova explosions. After the supernova explosion the ejected material 
drives a (supersonic) blast wave into the ambient medium to produce a supernova remnant. The interaction of the velocity field produced by the supernova with the surrounding magnetic field gives rise to a turbulent electromotive force $\mathbf{E}_{\text {turb }}=\left\langle\mathbf{u}^{\prime} \times \mathbf{B}^{\prime}\right\rangle$, which can be expressed in terms of the $\alpha$-effect and an additional magnetic diffusion due to the turbulent motions as follows:

$$
E_{i}=\alpha_{i j}<B_{j}>+\beta_{i j k} \frac{\partial<B_{j}>}{\partial x_{k}} .
$$

We study the non-linear evolution of an isolated supernova explosion with a two-dimensional, axisymmetric computer code in cylindrical coordinates. The magnetic field is assumed to be uniform and purely vertical (i.e. parallel to the rotation axis). Therefore, only the $\alpha_{z z}$ component of the $\alpha$-tensor can be obtained with this method:

$$
\bar{\alpha}_{z z}(z)=\frac{\left\langle V_{r}^{\prime} B_{\varphi}^{\prime}-V_{\varphi}^{\prime} B_{r}^{\prime}>\right.}{<B_{z}>}=\frac{\int\left(V_{r}^{\prime} B_{\varphi}^{\prime}-V_{\varphi}^{\prime} B_{r}^{\prime}\right) r d r d t}{\int B_{z} r d r d t}
$$

We apply these results to an ensemble of supernova explosions by specifying the spatial distribution of explosions:

$$
\alpha(z)=\int \phi\left(z_{0}\right) \cdot \bar{\alpha}_{z z}\left(z-z_{0}\right) d z_{0}
$$

where $\phi\left(z_{0}\right)$ and $z_{0}$ are the supernova rate per volume and the site of the explosion on the rotation axis, respectively.

\section{Basic equations and numerical methods}

We made the following assumptions for our numerical simulations: (1) the magnetic field is frozen in the gas, (2) the magnetic energy is small compared to the kinetic energy of the flow; therefore, the back reaction of the magnetic field on the dynamics is not taken into account, $(3)$ the gas rotates with constant angular velocity $\Omega_{0}$, (4) the external gravitational potential has only $z$-dependence with a constant gravitational acceleration. Cylindrical coordinates $(r, \varphi, z)$ are adopted with the $z$-direction parallel to the rotation axis. Axial symmetry is assumed so that the evolution is two-dimensional with $\partial / \partial \varphi=0$, but the components of magnetic field and velocity in the azimuthal direction are non-zero. . We consider an isothermal gas. The initial density and pressure distributions are calculated by using the equation of state and the equations of hydrodynamic equilibrium. 
The supernova explosions are simulated by a sudden release of thermal energy within a small volume on the rotation axis. The energy released by each supernova explosion is assumed to be $10^{51} \mathrm{erg}$, the mass of the ejecta $5 M_{\odot}$.

In a typical run the $(\mathrm{r}, \mathrm{z})$ mesh consists of $(124 \times 124)$ uniformly distributed grid points providing a resolution of $\Delta z=\Delta r=1.65 \mathrm{pc}$. In all simulations we choose $T=10^{4} \mathrm{~K}$ for the temperature, $\mu=$ 1.6 for the mean molecular weight, $\rho_{0}=6.12 \cdot 10^{-25} \mathrm{~g} \mathrm{~cm}^{-3}$ for the density at the galactic center, and $\Omega_{0}=40 \mathrm{~km} / \mathrm{s} / \mathrm{kpc}$ for the galactic rotation. Axial symmetry with respect to the $r=0$ axis and mirror symmetry with respect to the $z=0$ plane are assumed. We use free (open) boundaries for $r=r_{\max }$ and $z=z_{\max }$.

The equations of hydrodynamics are solved with an explicit second order scheme described by Różycka(1985). The magnetohydrodynamic field transport (induction) equations are solved with the so-called constraint transport (Stone and Norman, 1992).

\section{Results of the numerical simulations}

\subsection{SN EXPLOSION IN A UNIFORM MEDIUM}

We first consider the expansion of an isolated axisymmetric explosion in a uniform ambient medium. The supernova is assumed to explode in the galactic center. Qualitatively, the evolution of the supernova remnant proceeds as described by Chevalier(1974). A structure characteristic of adiabatic explosions (Sedov solution) is quickly achieved, with about $1 / 3 \cdot E_{S N}$ converted into kinetic energy and $2 / 3 \cdot E_{S N}$ remaining as thermal energy. Since the magnetic field is frozen in the gas the propagating blast wave sweeps up the surrounding magnetic field into the dense shell of the supernova remnant.

We determine from our numerical simulations the $\alpha_{z z}$ for a single explosion (eq.(1)) and derive from these results the $\alpha$-effect due to an ensemble of explosions by specifying the supernova distribution function (eq.(2)). We only consider the case that the explosion rate varies with distance from the galactic midplane and assume that supernovae are distributed according to

$$
\phi\left(z_{0}\right)=\frac{\sigma}{\sqrt{2 \pi} \cdot H_{\sigma}} \cdot \exp \left(-z_{0}^{2} / 2 H_{\sigma}^{2}\right)
$$

where $\sigma$ is the supernova rate per unit area in the galactic midplane, $H_{\sigma}$ is the scale height of the explosion rate, and $z_{0}$ is the location of the explosion above the galactic midplane.

Figure 1 shows the $\alpha_{z z}$-profile derived with the distribution function $\phi\left(z_{0}\right)$ for scale heights $H_{\sigma}=0,50,100$, and $200 \mathrm{pc}$. The case $H_{\sigma}=0$ and $z_{0}=0$ gives $\phi\left(z_{0}\right)=\sigma \cdot \delta(0)$ and corresponds to the situation that 
all supernovae explode only in the galactic plane. Then, the resulting $\alpha$-distribution due to an ensemble of explosions is identical to the case of a single explosion. With increasing scale height $H_{\sigma}$ the amplitude of the $\alpha$-parameter becomes smaller while the width of its $z$-profile becomes larger. The amplitude decreases as a result of the partial cancelation due to explosions above and below the galactic midplane. The maximum of $\alpha$ is $60 \mathrm{~m} / \mathrm{s}$ for $H_{\sigma}=0$, and $30 \mathrm{~m} / \mathrm{s}, 15 \mathrm{~m} / \mathrm{s}$, and $5 \mathrm{~m} / \mathrm{s}$ for $H_{\sigma}=50,100$, and $200 \mathrm{pc}$, respectively. For explosions located in the galactic plane, $\alpha_{z z}$ is negative in the northern hemisphere and positive in the southern hemisphere.

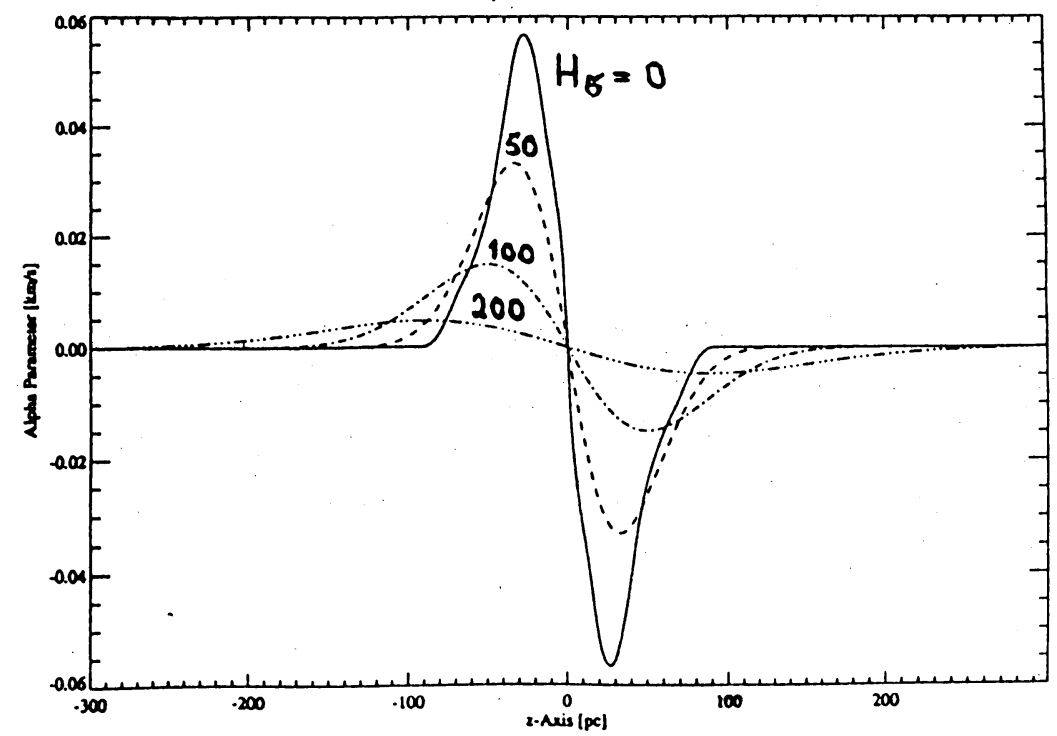

Fig. 1: The $\alpha_{z z}$-profile for different scale heights of $\phi\left(z_{0}\right)$.

\subsection{SN EXPLOSION IN A STRATIFIED MEDIUM}

We now consider the evolution of an isolated supernova explosion in a stratified medium $\rho=\rho_{0} \cdot \exp \left(-z / H_{\rho}\right)$, with the density scale height $H_{\rho}$. Numerically, we study the propagation of the supernova remnant in a medium with $H_{\rho}=100,300$, and 600 pc. In Fig. 2 we show the $\alpha_{z z}$-profile (averaged over time and radius) derived from the numerical simulations with $H_{\rho}=100 \mathrm{pc}$ for a single explosion: We again find negative values for $z>z_{0}$ and positive values for $z<z_{0}$. The asymmetry of the $z$-profile is due to the stratification. This asymmetry determines $\alpha_{z z}$ due to an ensemble of explosions (eq.(2)). Figure 3 shows the $\alpha$-values derived from the numerical simulations for the extreme case of uniform explosion rate for different density scale heights. The resulting $\alpha_{z z}$-values are spatially uniform except for a change in sign at the midplane. The influence of the density distribution proved to be small. We find $\alpha$-values of only few meters 
per second for a density scale height of $100 \mathrm{pc}$ and even smaller values for larger density scale heights.

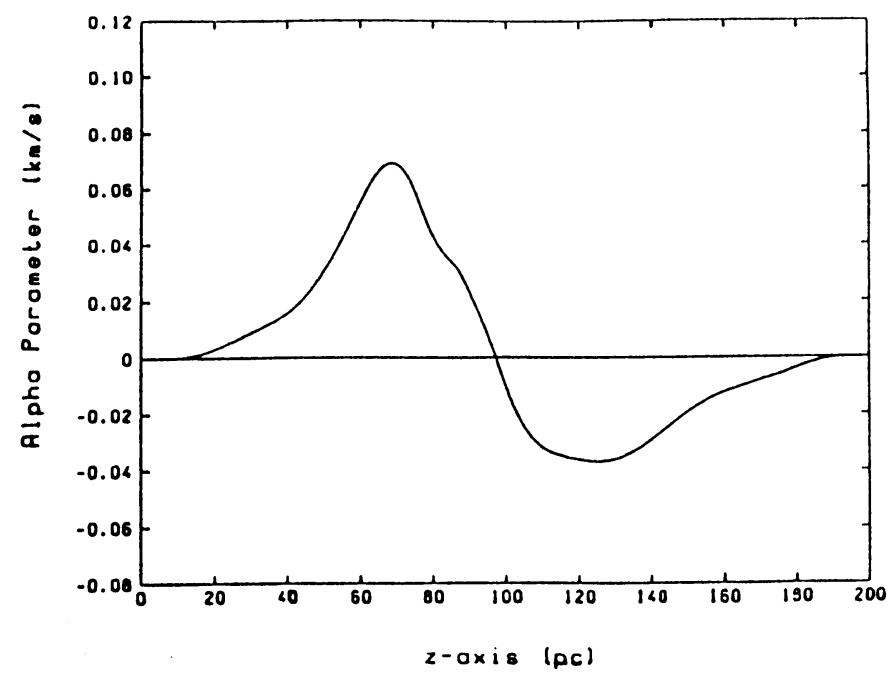

Fig.2: The $\alpha_{z z}$-profile in a stratified medium with $H_{\rho}=100 \mathrm{pc}$.

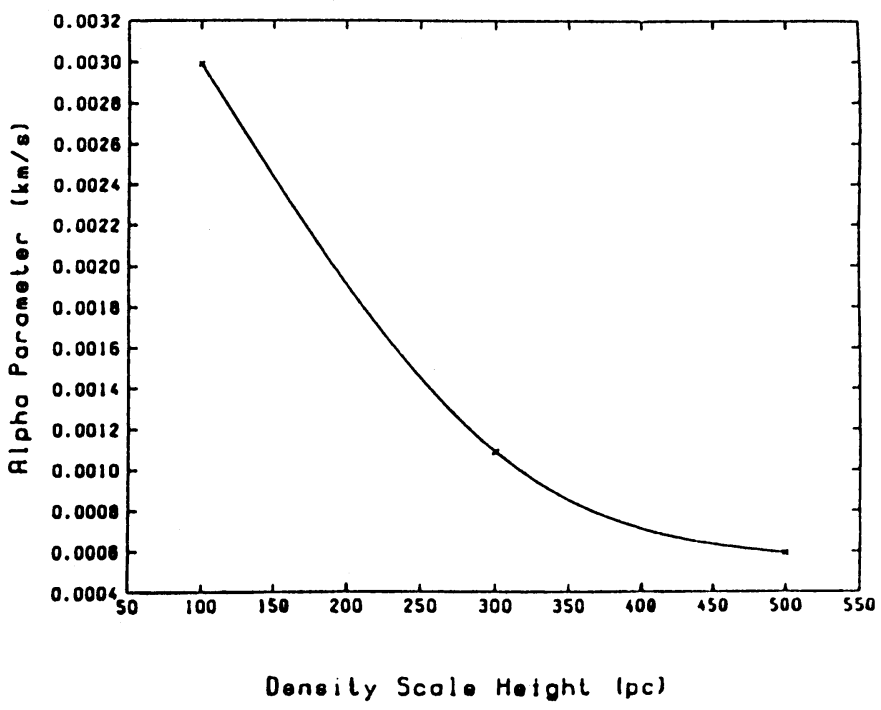

Fig. 3: The $\alpha_{z z}$-values for different density scale heights.

\section{References}

Chevalier, R.A. 1974, Ap.J., 188, 501.

Różyczka, M. 1985, Astron. Astrophys., 143, 59.

Stone, J.M., and Norman, M.L. 1992, Ap.J. Suppl., 80, 791. 